\title{
Science réglementaire et démocratie technique
}

\section{Réflexion à partir de la gestion des pénuries d'eau}

\author{
Rémi Barbier ${ }^{1}$, Jeanne Riaux², Olivier Barreteau ${ }^{3}$ \\ 1 Sociologue, ENGEES, UMR Gestion des services publics, 1 quai Koch, BP 61039, 67070 Strasbourg cedex, France \\ 2 Anthropologue, IRD, UMR G-EAU, Maison des sciences de l'eau, 300 avenue Émile Jeanbrau, BP 64501, \\ 34394 Montpellier cedex 5, France \\ 3 Sciences de l'eau, CEMAGREF, UMR G-EAU, 361 rue Jean-François Breton, BP 5095, 34196 Montpellier cedex, France
}

\begin{abstract}
La gestion des pénuries d'eau renvoie à un cadre d'action publique qui suppose la mise en place d'instruments de mesure pour qualifier les situations hydrologiques. Les auteurs, au croisement de l'anthropologie des techniques et des science studies, proposent deux concepts-clés susceptibles d'éclairer les processus sociotechniques en jeu dans la construction de rapports entre la connaissance et l'action. La science réglementaire d'une part, qui correspond à un travail de production de connaissances de façon à en faire des instruments d'action et de régulation. La chaîne opératoire de l'autre, qui permet de reconstituer les interactions entre opérations techniques et agents humains dans l'émergence de la science réglementaire. Cette approche de sciences sociales sensible aux objets et aux techniques convoque de nombreux points de vue disciplinaires avec pour enjeu, une véritable démocratie technique.
\end{abstract}

La Rédaction

\section{Mots-clés :}

pénurie d'eau ; anthropologie des techniques ; sociologie des sciences : chaîne opératoire ; France

\section{Keywords:} water scarcity; anthropology of techniques; social science studies; operational chain; France

\begin{abstract}
Résumé - L'infrastructure sociotechnique nécessaire à la mise en œuvre des politiques environnementales recèle un véritable contenu politique : elle cadre et influence l'orientation et les effets de l'action publique, souvent de manière implicite. Une perspective normative de gouvernance démocratique demande de la rendre discutable. Nous abordons les problématiques relatives à cette mise en discussion dans le cas de la gestion des pénuries d'eau en France. Dans un premier temps, nous décrivons la mise en place de cette infrastructure et qualifions cette activité en mobilisant le concept de science réglementaire. Nous identifions ensuite les formes de critique mobilisées par les acteurs de l'eau au sein des comités sécheresse. Nous proposons enfin un outil, la chaîne opératoire, pour rendre visibles et discutables la structure et le fonctionnement de l'infrastructure, et plus généralement la science réglementaire dont elle est issue. Cet outil permet de dépasser les formes de critique observées et de prendre en charge collectivement la tension entre principe de justice et principe d'adaptation aux conditions locales.
\end{abstract}

\begin{abstract}
Regulatory science and technical democracy. A reflection based on water shortage management in France. The sociotechnical infrastructure required to implement environmental policies has a definite political content: it frames and influences public action, most often in an implicit way. In this paper we take a normative stance on democratic governance, which raises the need for making this infrastructure debatable. We focus on the specific case of drought management in France, which has developed an infrastructure that consists of zoning, measurement networks and threshold definition for critical flows in order to be able to assess a current hydrological situation in any place and to associate it to a specific crisis level. First we identify the various criticisms voiced by stakeholders in drought committees such as challenging the infrastructure's technical validity, setting out its inadequacy to a specific context,
\end{abstract}

Auteur correspondant : J. Riaux, jeanne.riaux@ird.fr

Les deux premiers auteurs sont mentionnés par ordre alphabétique. 
disqualifying its use due to the gap between science and the existing practice. We then suggest introducing a tool borrowed from the anthropology of techniques : the operational chain. We propose to use it to render explicit and debatable the infrastructure, its structure and content and on top of that the "regulatory science" which it stems from. This tool entails overcoming the various forms of criticism observed as well as dealing collectively with the tension between principles of justice and adaptation to local conditions. We argue that it may also provide a medium to associate lay people in the development or the revision of the infrastructure itself.

\section{Introduction}

L'action publique ne saurait être réduite à des confrontations d'idées, des luttes d'institutions ou des conflits $\mathrm{d}^{\prime}$ intérêts ${ }^{1}$. L'équipement technique et cognitif y joue également un rôle déterminant. La notion d'instrumentation de l'action publique a été proposée par Lascoumes et Le Galès (2004) pour problématiser le choix et l'usage des outils permettant de matérialiser et de rendre opérationnelle l'action gouvernementale. Pour compléter cette approche, Le Bourhis (2004) propose la notion d'infrastructure d'action publique, définie comme l'« ensemble des dispositifs cognitifs et organisationnels qui interviennent dans la production des actions publiques ", notamment sous la forme de réseaux sociotechniques. Ces travaux invitent à considérer l'action publique sous l'angle du façonnage de cette infrastructure, avant qu'elle ne soit stabilisée puis transformée, plus ou moins consciemment, par ses producteurs en «boîte noire » à l'aspect lisse et à l'apparence objective (Riaux, à paraître). L'enjeu est majeur dans la mesure où cette infrastructure participe largement à la délimitation du champ des questions possibles, des problèmes traitables et à la construction des réponses. C'est notamment le cas pour la gestion publique de l'environnement, et plus précisément celle des pénuries d'eau qui nous occupe ici. Le déclenchement éventuel de mesures de restriction des usages de l'eau repose sur un ensemble d'outils techniques (zonages, seuils hydrologiques, instruments de jaugeage) qui ont pour rôle essentiel de qualifier la criticité de la situation hydrologique. L'infrastructure sociotechnique de l'action publique a donc ici un rôle central dans la décision associée à la gestion des pénuries.

À partir de ces constats, notre réflexion s'articule autour de deux ordres de questionnement ${ }^{2}$. Du point de vue de l'analyse, que se passe-t-il lorsque cette infrastructure, généralement discrète, voire implicite, se trouve sur le devant de la scène, soit qu'il y ait controverse, soit

\footnotetext{
${ }^{1}$ Ce texte s'appuie sur des travaux réalisés dans le cadre de la convention de recherche OnEma-Cemagref.

${ }^{2}$ Ce travail repose sur une série d'enquêtes approfondies auprès d'acteurs de l'eau, ainsi que sur le suivi des comités sécheresse de deux départements entre juin 2007 et juin 2008 dans le cadre d'une recherche post-doctorale au Cemagref conduite par J. Riaux. Une trentaine d'entretiens ont été réalisés et leur exploitation a été enrichie par l'observation directe des réunions du comité sécheresse de l'un des départements, ainsi que par le recueil et l'analyse d'une abondante littérature grise (comptes rendus de réunions, notes de travail...).
}

que cela résulte de l'exigence participative de l'action publique contemporaine? Nous verrons que la mise en discussion des modalités de gestion des pénuries peut notamment se solder par une mise en cause de la légitimité de l'infrastructure à dire la vérité sur les situations hydrologiques. Du point de vue de l'action, comment mettre en œuvre une discussion, voire une coconstruction de cette infrastructure complexe, qui puisse en consolider la légitimité en temps de crise?

Nous présentons tout d'abord le dispositif de gestion des pénuries d'eau en France et soulignons la place $q^{\prime}$ 'y occupe l'infrastructure sociotechnique. Puis nous exposons les formes de critique de cette infrastructure par les acteurs des comités sécheresse. Enfin, nous proposons un outil : la chaîne opératoire, qui représente un moyen potentiellement efficace pour rendre visibles et discutables la structure et le fonctionnement de l'infrastructure en dépassant les formes de critique observées.

\section{«Sommes-nous en crise? » Le travail de qualification de la science réglementaire}

\section{La gestion des pénuries : une vue d'ensemble}

Pris en application de la loi sur l'eau de 1992, le décret $n^{\circ}$ 92-1041 relatif à la limitation et à la suspension provisoire des usages de l'eau, communément appelé « décret sécheresse », prévoit la mise en place d'un dispositif d'anticipation et de gestion des situations de pénurie d'eau. C'est la succession des sécheresses en 2003, 2005 et 2006 qui a contribué à faire de ce problème un enjeu national ${ }^{3}$ et conduit à la généralisation, à l'échelon départemental, de plans d'action sécheresse dont l'objectif est double : prévoir les périodes de tension sur la ressource en eau ; proposer des règles d'action visant à limiter les conséquences d'une crise potentielle ou à en prévenir l'occurrence. Ce dispositif articule trois acteurs majeurs : une autorité administrative habilitée à promulguer un arrêté de restriction des usages de l'eau (le préfet); un comité sécheresse, arène regroupant les différents acteurs

\footnotetext{
${ }^{3}$ Une circulaire ministérielle, «Plan d'action sécheresse », a été diffusée le 30 mars 2004 et un «Plan de gestion de la rareté de l'eau », présenté en Conseil des ministres le 26 octobre 2005.
} 


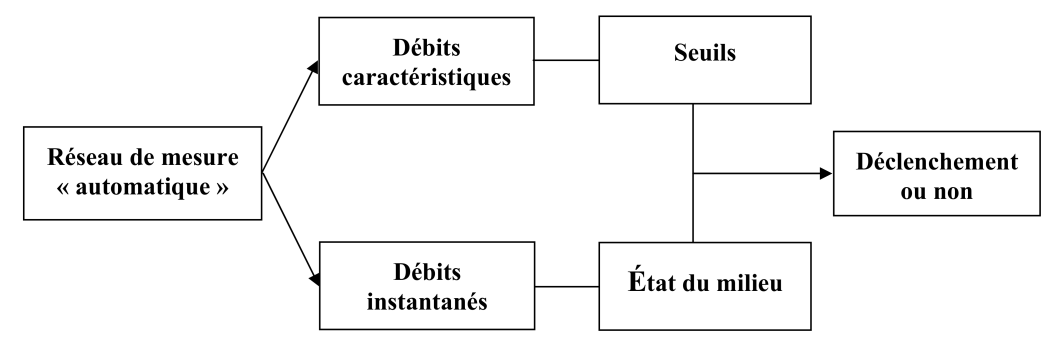

Fig. 1. Format réglementaire de l'infrastructure de gestion des pénuries d'eau.

de l'eau du département (administrations ${ }^{4}$, représentants des usagers de l'eau, élus, associations, etc.) et au sein de laquelle sont discutés, à froid, le dispositif proposé par l'administration et, à chaud, la réalité de la situation, les mesures à prendre et les éventuelles dérogations ; l'infrastructure, enfin, qui permet de déterminer si l'on est en crise et quel est le degré de gravité de la situation. Les compositions de l'arène et de l'infrastructure sont propres à chaque département.

Cette infrastructure comprend elle-même plusieurs éléments articulés entre eux (Fig. 1) ${ }^{5}$. Le principe général en est le suivant. Un réseau de mesure hydrologique produit des données sur l'état d'un cours d'eau. Ces données sont utilisées pour produire des valeurs caractéristiques (ici des débits) sur lesquelles repose généralement la définition de seuils hydrologiques. Ces seuils sont associés à des classes d'état du milieu (vigilance, alerte, crise) ; le franchissement d'un seuil marque le passage d'une classe d'état à une autre. Les données permettent en parallèle d'informer en temps réel sur l'état du milieu. La comparaison d'une valeur instantanée aux seuils permet alors d'identifier un franchissement et de déclencher ou non des restrictions. Celles-ci doivent être appliquées dès la parution de l'arrêté préfectoral notifiant le passage du seuil.

L'ensemble du dispositif est cadré par la réglementation. Mais, alors que la déclinaison des mesures de restriction possibles est bien détaillée dans les textes, la réglementation demeure plutôt silencieuse sur la manière de construire l'infrastructure sociotechnique. Aux questions relatives à l'identification d'un réseau de mesure pertinent à l'étiage, à la manière de déterminer un seuil hydrologique d'alerte ou de crise, etc., chaque mission interservices de l'eau (MISE) - en charge des plans sécheresse pour le compte du préfet - doit apporter ses propres réponses, en fonction du contexte local et des

\footnotetext{
${ }^{4}$ Les administrations les plus impliquées dans la gestion des pénuries sont les directions départementales de l'agriculture et de la forêt (DDAF), les missions interservices de l'eau (MISE) et les directions régionales de l'environnement (DIREN). L'Office national de l'eau et des milieux aquatiques (ONEMA) a aussi une place centrale au sein du dispositif.

${ }^{5}$ On trouvera une description plus détaillée, à partir de deux cas d'étude, de cette infrastructure et du dispositif réglementaire dont elle émane dans Riaux (2008).
}

moyens à disposition. En conséquence, chaque plan sécheresse départemental et, plus encore, chaque infrastructure d'évaluation et de déclenchement revêtent une forme originale.

L'absence de cadrage plus précis s'explique simplement. D'une part, il n'existe pas de savoirs stabilisés et universellement valides sur les conditions de construction d'un réseau de mesure apte à dire la réalité hydrologique ou sur la qualification objective de situations de «crise » ou «d'alerte ». D'autre part, l'hétérogénéité des situations locales, du triple point de vue de l'hydrologie, de la disponibilité de données et de la sensibilité des acteurs aux mesures de restriction, impose de maintenir ouvertes des marges d'adaptation pour les acteurs locaux. De ce fait, sur le terrain, la construction de l'infrastructure procède de tâtonnements, d'ajustements et de recyclages d'objets techniques et de connaissances préexistants. Il en résulte un ensemble complexe, s'adaptant au fur et à mesure des expériences et de l'évolution des usages et des connaissances. La section suivante est consacrée à l'illustration de ces difficultés sur les trois principales composantes de l'infrastructure, à savoir le zonage hydrographique, les réseaux de mesure et les seuils.

\section{L'infrastructure d'évaluation des situations hydrologiques}

L'anticipation et la gestion de la crise impliquent en premier lieu d'avoir déterminé des zones d'alerte ${ }^{6}$ au sein desquelles s'appliqueront le cas échéant des mesures différenciées. À cet égard, le bassin versant tend à s'affirmer comme le territoire légitime. Le découpage en zones d'alerte suit alors la cartographie de ces bassins, laquelle correspond en général aux cours d'eau structurants du réseau hydrographique départemental. Mais le zonage peut être négocié et affiné, à l'initiative des services de l'État et/ou à la demande des acteurs concernés. Ces ajustements sont justifiés par différents arguments : ne pas pénaliser tout un territoire du seul fait de l'existence d'un problème localisé dans une «zone d'étiage sensible »; faciliter l'application ultérieure des mesures, comme dans ce département où le découpage en sept zones est ajusté

\footnotetext{
${ }^{6}$ Le décret $\mathrm{n}^{\circ} 92-1041$ parle $\mathrm{d}^{\prime}$ « unités hydrographiques cohérentes ».
} 
à la perspective d'organisation d'un cycle hebdomadaire de tours d'eau ; tenir compte de la situation particulière des têtes de bassin versant ou de certaines zones réalimentées par des retenues artificielles. Le risque est alors de nourrir le sentiment d'injustice par le constat qu'il existe des «distorsions aux frontières », et de contrevenir à cette exigence morale largement répandue selon laquelle, "en période de crise, tout le monde doit faire des efforts ». L'administration doit ainsi gérer une tension entre deux principes : justice entre usagers et adaptation aux contingences locales.

Deuxième composante de l'infrastructure, les réseaux de surveillance sont constitués de stations débitmétriques et/ou de forages piézométriques qui mesurent respectivement les niveaux des cours d'eau et ceux des nappes souterraines. Au regard des caractéristiques hydrogéologiques locales, et notamment des liens entre rivières et nappes, une première question pourra se poser : l'état de la ressource sera-t-il mieux saisi à travers les débits des cours d'eau, plus sensibles aux pluies et au ruissellement du bassin versant, ou à travers le niveau des nappes? En fonction de la réponse, la sensibilité aux événements pluvieux sera plus ou moins prise en compte, favorisant certains usagers au détriment d'autres. Dans un département, il fut ainsi décidé d'opter pour les stations débitmétriques, pourtant jugées techniquement moins pertinentes ; mais le souci de ne pas pénaliser les agriculteurs locaux par rapport à ceux du département voisin, où ce choix avait été fait, prévalut. Par ailleurs, les réseaux mobilisent généralement des stations de mesure qui leur préexistent. Leur fonction originelle concerne plutôt la prévention des risques de crue ou la régulation hydraulique des barrages. Ces stations se révèlent alors plus ou moins adaptées à la mesure des débits d'étiage, qu'il s'agisse de leur position sur le territoire ${ }^{7}$ ou de leur fonctionnement. La capacité des valeurs produites à qualifier l'état hydrologique de l'ensemble de la zone d'alerte peut aussi être mise en question : une station située, par exemple, en aval du cours d'eau principal peut-elle rendre compte de la situation des petits affluents, du « chevelu»? Le souci de représentativité se heurte ici au manque de moyens humains et financiers pour densifier le réseau.

Ces données doivent permettre d'effectuer deux opérations décisives : d'une part, le calcul des seuils, c'està-dire des valeurs déterminant le passage d'un état de la situation hydrologique à un autre (situation normale, de vigilance, de crise, de crise renforcée); d'autre part, la mesure en temps réel de la situation hydrologique qui permettra, par comparaison avec la valeur des seuils, de qualifier l'état des milieux. Les seuils sont - dans la

\footnotetext{
7 Une localisation en aval du bassin ou à la confluence de gros émissaires permet aux stations de jaugeage de bénéficier des apports du ruissellement sur l'ensemble du bassin versant. Dès lors, la représentativité de leurs résultats sera contestée par les défenseurs du milieu.
}

plupart des cas - définis à partir de "valeurs caractéristiques » qui sont souvent elles-mêmes produites dans le cadre d'autres objectifs réglementaires. En effet, comme l'a montré Lascoumes (1994), toute nouvelle politique vient se surajouter aux programmes d'action antérieurs, héritant et recyclant des catégories de pensée, d'action ou de jugement et, pour ce qui nous concerne, des éléments infrastructurels produits à d'autres fins. Ainsi, le « débit quinquennal sec » (QMNA5) est mobilisé pour les régimes de déclaration ou d'autorisation des prélèvements; de son côté, le " $10^{\mathrm{e}} \mathrm{du}$ module » a été associé à partir de 1984 au débit minimum biologique à laisser dans les cours $\mathrm{d}^{\prime} \mathrm{eau}^{8}$. Ces valeurs caractéristiques sont mobilisées pour le calcul des seuils... en grande partie parce qu'elles ont le mérite d'exister ${ }^{9}$. Leur détermination présuppose toutefois l'existence de chroniques de données suffisamment longues, qui confèrent leur robustesse aux opérations statistiques sous-jacentes. À défaut, les données de base et/ou les valeurs caractéristiques elles-mêmes devront être obtenues autrement (cf. infra).

Les données produites sur le terrain permettent par ailleurs de déterminer l'état «instantané » du milieu (il y a $x \mathrm{l} / \mathrm{s}$ dans telle rivière, à tel moment, à tel endroit) ; souvent, elles seront en fait retravaillées et lissées en fonction d'un pas de temps précédemment défini, par exemple une moyenne glissante sur trois ou cinq jours consécutifs. Pour compléter l'infrastructure, il ne reste plus alors qu'à établir la règle en fonction de laquelle la mise en rapport des seuils et des valeurs «instantanées » enclenchera un changement de qualification de l'état du milieu : suffitil, par exemple, que la valeur instantanée passe sous le seuil, ou ce constat doit-il être répété deux ou trois fois de suite? La réponse dépasse la portée de la science hydrologique. Elle intégrera notamment des impératifs pratiques simples : éviter une trop grande fréquence de prise et de levée d'arrêtés et éviter des changements trop fréquents dans les mesures de restriction.

\footnotetext{
${ }^{8}$ La question du « débit réservé » illustre bien les pratiques de l'administration en matière de construction de l'infrastructure. La loi de 1984 impose de respecter un débit minimum permettant la protection des milieux aquatiques. Le mode de calcul de ce débit réservé doit être propre à chaque cours d'eau. Mais, en l'absence de connaissance suffisante des milieux, la plupart des départements ont fixé ce débit au $10^{\mathrm{e}}$ du module, dont le mode de calcul est accessible car fondé sur des indicateurs hydrologiques déjà identifiés (contrairement aux indicateurs biologiques). Cette valeur statistique a été produite pour de nombreux cours d'eau. Elle est alors « recyclée » dans d'autres cas, stabilisée comme valeur réglementaire «solide».

9 C'est ainsi que, dans un département, le seuil de crise a été défini par l'hydrologue de la DIREN comme la moyenne du QMNA5 et du $10^{\mathrm{e}} \mathrm{du}$ module. Ce mode de calcul résulte d'un test rétrospectif sur une année de référence : il s'agissait de simuler les effets de l'adoption de différentes valeurs pour les seuils (date d'entrée en crise et conséquences pour l'agriculture locale) et d'en évaluer l'acceptabilité.
} 
Les limites des indicateurs, en termes de représentativité spatiale et temporelle, ainsi que la complexité de calcul des valeurs de référence ouvrent la porte à une négociation à chaque fois qu'une application simple des mesures semblerait justifiée. "Ces niveaux contribuent au processus de prise de décision, mais sont également grandement nuancés en cellule sécheresse ", reconnaît un agent de MISE. En définitive, ce façonnage progressif de l'infrastructure apparaît comme une activité hybride, mêlant des éléments de science hydrologique et de métrologie, des legs de politiques plus anciennes, des ajustements aux données disponibles et des négociations dont l'horizon régulateur est celui de la prise de décision, laquelle intègre notamment des anticipations en matière d'acceptabilité par les usagers. Cette pratique s'apparente bien à ce que Jasanoff $(1990$, p. 229) appelle science réglementaire, «a hybrid activity that combines elements of scientific evidence and reasoning with large doses of social and political judgement ». La science réglementaire est soumise à des contraintes spécifiques qui, sans évidemment l'invalider, la distinguent de la science académique. Comme l'explique avec force un acteur, «ça doit coller au terrain, être crédible, compréhensible, avoir de la souplesse».

Pour autant, la tentation est grande de masquer cette différence et de transformer l'infrastructure en boîte noire indiscutable parce que "scientifique». "Une fois les seuils définis entre experts - explique un hydrologue de la DIREN -, on les présente en comités sécheresse, qui sont plus politiques puisque regroupant des usagers. Là, on présente les résultats, les conclusions. Au sein de ces comités, on ne rentre pas dans le détail de toute la discussion technique qu'on a pu avoir. Par souci de simplification et pour ne pas polluer le débat, on dit que les seuils sont calculés mathématiquement, statistiquement, sans entrer dans le détail des calculs. » Ailleurs, alors qu'une controverse relative au choix des seuils mobilise à plein temps les administrations concernées (DIREN de bassin, DIREN et DDAF), le choix est fait de ne pas rendre public le problème et de ne pas l'évoquer en comité sécheresse. Il s'agit clairement de soustraire l'infrastructure à l'épreuve de la critique et de limiter ainsi les discussions dans les comités sécheresse à des négociations d'adaptation à caractère plus ou moins dérogatoire (retarder de quelques jours la promulgation de l'arrêté, par exemple).

\section{La mise en discussion de l'infrastructure sociotechnique}

\section{L'infrastructure mise à l'épreuve}

Ce mode de fonctionnement est-il tenable? L'infrastructure peut-elle être soustraite longtemps à l'épreuve de la publicité? Dans une certaine mesure, cette situation peut convenir aux protagonistes, au moins pour un temps. C'est ce que l'on a observé, par exemple, dans un département à propos de la définition d'un seuil de crise renforcée susceptible d'entraîner une interdiction totale d'irriguer. La chambre d'agriculture avait décidé de ne pas s'impliquer dans la discussion afin de ne pas perdre sa légitimité auprès des agriculteurs en cas d'adoption de ce niveau de restriction, mais aussi pour se réserver une possibilité de le contester ultérieurement. Mais les enjeux associés aux mesures de restriction sont parfois tels qu'ils finissent par mettre en branle une dynamique de controverse, au cours de laquelle les acteurs sont finalement amenés à s'intéresser à la boîte noire de l'infrastructure, à mettre en lumière ces éléments jusqu'alors invisibles et dont la robustesse n'était pas nécessairement discutée. Que se passe-t-il alors dans ces situations ? En première approche, trois formes de mise à l'épreuve critique de l'infrastructure peuvent être analytiquement distinguées, correspondant à ce qu'on peut appeler des pratiques ordinaires de critique technique : la contestation, le contournement et la disqualification (Barbier, 2005).

Ce que nous qualifions de contestation correspond à une mise en cause technique de l'infrastructure au vu des résultats qu'elle produit, qui ne sont pas reconnus comme valides. Pour être entendue, sinon acceptée, cette contestation doit naturellement être justifiée. Il s'agit ici de produire des jugements alternatifs sur l'état de la situation hydrologique. Une première modalité de contestation repose sur la mise en avant d'un décalage entre le « dit » de l'infrastructure et la situation vécue par les acteurs de l'eau. Elle oppose des formats de connaissance alternatifs à celui produit par le centre de calcul de l'administration. Il s'agit par exemple de celui de certains agriculteurs, ancré dans un rapport au monde personnel et quotidien : " L'infrastructure dit qu'on est en crise, alors que j'ai de l'eau dans mon puits ", ou de celui des agents de l'ONEMA, qui, grâce à leur présence sur le terrain, peuvent constater un nombre important d'assecs sur le réseau de chevelus, alors que l'infrastructure, construite autour des cours d'eau plus importants, ne signale pas encore de tensions sur la ressource. La prise en compte de ces divers formats de connaissance dans le dispositif de gestion des pénuries permet le cas échéant d'absorber cette critique dans une logique intégrative. Le second levier de contestation, qui implique une plus grande familiarité avec le dispositif, interroge plus directement la pertinence de certains choix techniques et tend vers la contre-expertise. Ainsi, dans un département, la profession agricole procéda à des contre-mesures de débits, mettant ainsi en doute l'exactitude des mesures proposées par l'administration. Au cours de ce travail de recueil de données, la profession agricole s'érigea progressivement en partenaire à même de négocier avec l'administration la mise en place d'un réseau de mesure alternatif. Après plusieurs années de discussions, d'essais, de jaugeages et de contre-jaugeages, 
les points de mesure négociés furent validés et fixés sur un document cartographique. Dans ce cas, la contestation se solde par une reconstruction progressive du réseau de mesure, qui acquiert ainsi une légitimité auprès des membres du comité sécheresse.

La seconde forme de réception critique, le contournement, correspond à une tentative de désactivation de l'infrastructure : il ne s'agit pas de contester tel ou tel résultat, mais d'établir tout simplement que l'infrastructure ne peut ou ne doit pas servir à orienter la prise de décision. L'invocation de "clauses d'exception », relatives au milieu ou au contexte socioéconomique, semble jouer un rôle décisif à cet effet. Ainsi, en zone méditerranéenne, le contenu de l'infrastructure serait inadapté au « régime hydrologique méditerranéen » : «Nos rivières sont naturellement hors la loi! » estiment les chambres d'agriculture, parfois approuvées par d'autres membres des comités sécheresse, y compris des porte-parole du milieu tels que des animateurs de bassin versant. Le contournement peut également se rapporter aux conséquences du recours à l'infrastructure. Celle-ci produit en effet un point de vue sur la réalité (« on est en crise de niveau 2 ») qui entraîne la mise en œuvre de mesures de restriction contraignantes pour les usagers de l'eau. Dans certains cas, ces contraintes sont jugées inacceptables ou trop lourdes : leur mise en application est alors refusée ou négociée. Au fond, ce qui doit primer est moins la qualification objective de la situation hydrologique que la vulnérabilité respective des acteurs ou du milieu aux mesures. À cet égard, la mise en avant d'une exception humaine ${ }^{10}$ - «On ne peut tout de même pas favoriser la survie des poissons pendant que les agriculteurs sont en difficulté pour survivre »- et d'une résilience de la nature sont des ressources stratégiques utilisées par la profession agricole. L'explicitation et la mise en débat des vulnérabilités peuvent permettre de sortir de cette opposition en bloc pour argumenter dans le détail de telle légitimité ou de telle vulnérabilité. On n'aboutit pas nécessairement à un consensus, mais au moins à un dissensus bien posé (Riaux et al., 2009).

D'un point de vue normatif, la limite de ces deux premières formes de critique réside dans le risque de manipulation stratégique qui leur est sous-jacent : la prolongation artificielle de l'incertitude - sur la pertinence de certains choix techniques ou sur la vulnérabilité du milieu - peut participer d'une "stratégie de pouvoir au service de l'inaction environnementale » (Mermet, 2005). Toutefois, même si l'infrastructure est critiquée pour être incomplète, insuffisamment adaptée aux conditions locales, susceptible de conduire à des décisions perçues comme socialement inacceptables, sa capacité à peser sur la décision à prendre et le problème à régler ne lui est pas déniée par principe ; elle est simplement subordonnée à

\footnotetext{
${ }^{10}$ Qui renvoie à ce que Mermet (2002) qualifie de rhétorique
} pseudo-humaniste. une série d'ajustements. C'est ce qui distingue ces critiques de la troisième forme, la disqualification, qui correspond pour sa part à une démonétisation radicale de l'infrastructure et s'exprime volontiers sous la forme d'une sentence définitive : «c'est du bricolage!», «il n'existe pas de calcul objectif pour les seuils, c'est de la négociation ».... Ces jugements s'expliquent, selon nous, par le contraste entre la réalité de la «science réglementaire » telle qu'elle s'est finalement donnée à voir, précisément avec tous ses ajustements et compromis, et une norme externe qui lui servait en quelque sorte de paravent : en l'occurrence, les exigences de rigueur et d'exactitude habituellement associées à l'univers technoscientifique. Cela nous renvoie à la manière dont la science - qu'elle soit réglementaire ou non - est présentée aux non-scientifiques. De manière générale, alors qu'en laboratoire les marges d'incertitude sont des sujets de discussion et de réflexion centraux ${ }^{11}$, en dehors du monde de la science, ces incertitudes sont activement passées sous silence (Ha-Duong et al., 2007). Comme on l'a vu, les administrations rechignent à dévoiler les secrets de fabrication de l'infrastructure ; mais, lorsque ceux-ci sont découverts dans le cours de la controverse, le jugement est, en effet, sans appel : « C'est du bricolage! » Au demeurant, les agents de l'administration ne sont parfois pas loin eux-mêmes de partager cet avis, à l'image de cet ingénieur de DDAF déclarant en entretien : «[...] on a parfois l'impression que les chiffres sont tirés du chapeau. » Même si nos terrains n'ont pas permis de le mettre clairement en évidence, on peut faire l'hypothèse que, dans ce cas de figure, l'écueil résiderait moins dans la manipulation stratégique de l'incertitude que dans le rabattement dans un pur rapport de force affranchi de toute épreuve de réalité. Quoi qu'il en soit, au regard du double enjeu de l'effectivité et de la transparence de l'action publique, il convient de réfléchir aux modalités d'une mise en discussion de l'infrastructure sur un mode qui soit ajusté à sa nature de produit de la science réglementaire. Le potentiel que recèle à cette fin la notion de « chaîne opératoire » est exploré dans la partie suivante.

\section{Des chaînes opératoires pour rendre discutable l'infrastructure}

Une condition du traitement démocratique de l'infrastructure sociotechnique est de la rendre discutable sans pour autant lui faire perdre sa légitimité. Comment dévoiler les phases de négociations et d'arrangements, d'hybridations d'hydrologie et de savoirs non scientifiques, ainsi que les inévitables sources d'imprécision et d'incertitude qui caractérisent le fonctionnement de cette science réglementaire, sans la disqualifier pour autant?

\footnotetext{
11 La question de l'incertitude a fait l'objet d'un colloque de la Société hydrotechnique de France en 2008, tandis qu'une partie importante du Guide pratique d'hydrométrie édité par le Cemagref (2002) est consacrée à cette question.
} 


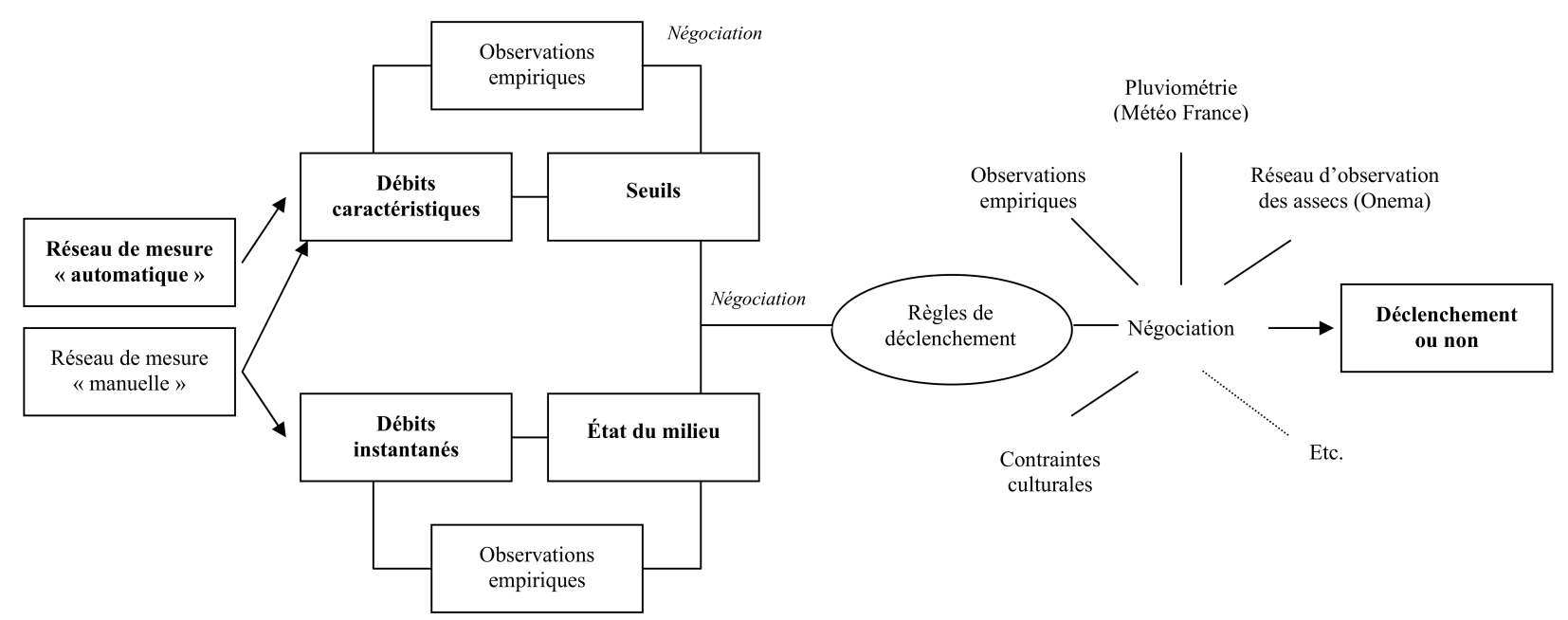

Fig. 2. Chaîne opératoire pour le déclenchement des arrêtés sécheresse.

Peut-être faut-il commencer par montrer que le «bricolage » dont elle semble résulter a du sens, qu'il ne rime pas avec arbitraire, mais qu'il est au contraire le moyen de prendre en charge la tension entre les exigences de justice et les exigences d'adaptation aux contingences locales. Il $\mathrm{s}^{\prime}$ agit alors de rendre explicite la construction de l'infrastructure, d'en ouvrir la boîte noire, pour en restituer le sens et la cohérence vis-à-vis de ses critiques. Un effort similaire visant à décrire les «instruments de visualisation » utiles à la gestion des inondations a été entrepris par Le Bourhis et Bayet (2002). Nous proposons pour notre part de mobiliser le concept de «chaîne opératoire ", qui permet de mettre en exergue les points sensibles de l'infrastructure sociotechnique et, à partir de là, de faire évoluer la manière de poser les problèmes qu'elle suscite.

Le concept de chaîne opératoire, issu à l'origine de la paléoanthropologie (Leroi-Gourhan, 1964), puis approprié par l'anthropologie des techniques (Lemonnier, 1980 ; Balfet, 1991 ; Cresswell, 1996), demeure relativement marginal aujourd'hui ${ }^{12}$. Il s'est pourtant imposé à nous, d'abord à des fins de compréhension, puis de description d'objets et de pratiques complexes : qu'est-ce qu'un seuil hydrologique? Comment calcule-t-on une valeur caractéristique telle que le QMNA5? Qu'est-ce qu'une station de jaugeage et qu'implique l'usage de tel instrument de mesure? Il fallait adopter une posture ethnographique permettant à la fois de suivre les processus de construction de l'infrastructure (étapes de construction du réseau de stations de mesure, par exemple), de mettre en relation l'histoire de chacun des éléments qui la compose (lien entre station de mesure et calcul des valeurs caractéristiques) et de rendre compte des variantes que peuvent

\footnotetext{
12 Si les chaînes opératoires demeurent en marge des travaux anthropologiques, elles n'en font pas moins l'objet de publications et de débats dans le champ de l'anthropologie des techniques, et notamment dans la revue Techniques et cultures.
}

choisir les constructeurs de cette infrastructure - ou qui s'imposent à eux.

Le concept de chaîne opératoire, à la fois descriptif et analytique, repose précisément sur la décomposition des activités techniques en une succession d'opérations reliées entre elles et permettant de suivre les étapes de fabrication d'un objet technique et d'identifier les processus techniques qui sous-tendent son existence. Cela permet également d'associer des personnes, des gestes et des matériaux aux procès de fabrication, donc d'observer le tissu d'interactions qui se façonne entre humains et nonhumains, dans l'action. Enfin, les modalités d'inscription graphique de la chaîne opératoire développées par les anthropologues des techniques se prêtaient à la restitution et à la mise en discussion du matériau ethnographique ainsi recueilli. Concernant l'infrastructure sociotechnique d'évaluation des situations de pénurie, deux niveaux d'observation et de formalisation sous forme de chaînes opératoires ont été retenus ${ }^{13}$.

Nous avons d'abord porté un regard englobant sur les étapes du processus d'évaluation de l'état des situations hydrologiques, en observant les pratiques concrètes des acteurs au sein et hors des comités sécheresse. Cela nous a permis de compléter la structure réglementaire du dispositif présentée précédemment (Fig. 1) en y ajoutant les opérations concrètes qui jalonnent les processus de décision. La figure 2 illustre les résultats de cette analyse.

Cette chaîne opératoire a été élaborée à partir de la situation d'un département dans lequel le plan d'action sécheresse s'appuie sur le débit des cours d'eau. Dans ce cas, les stations hydrométriques de la DIREN (automatiques)

\footnotetext{
13 Nous présentons ici le travail exploratoire entamé à propos des potentialités de cette démarche en matière de démocratisation de l'infrastructure de l'action publique. Une réflexion spécifique sur l'usage que nous faisons de la chaîne opératoire, à la lumière des avancées conceptuelles proposées par les anthropologues des techniques, sera réalisée ultérieurement.
} 


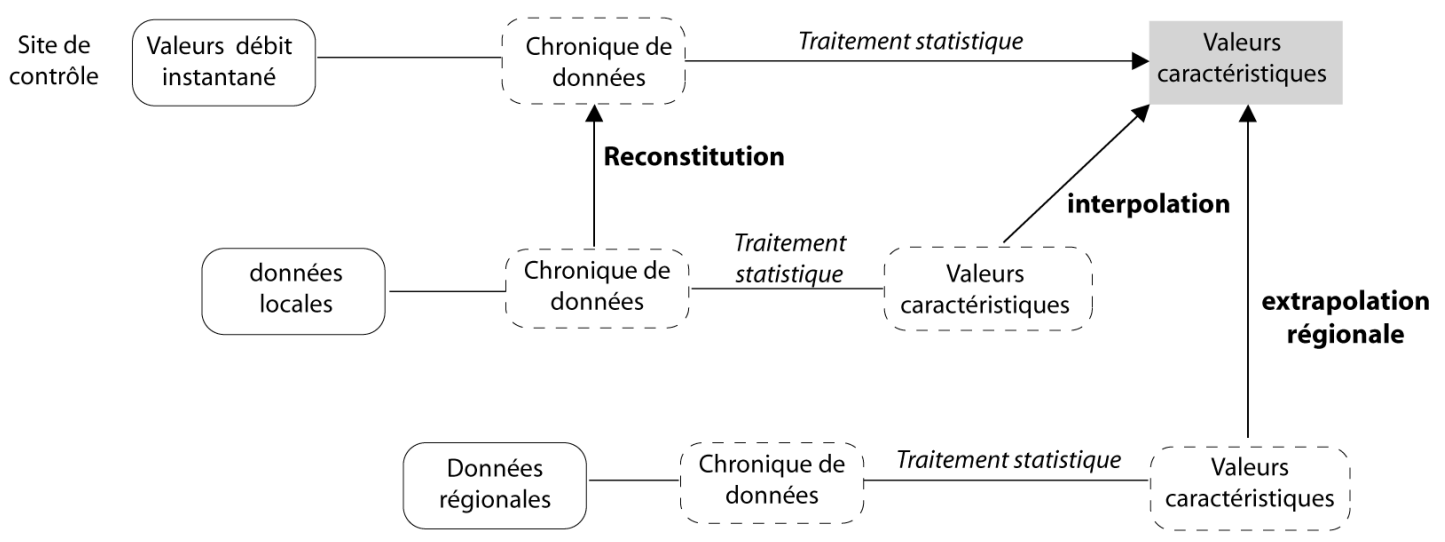

Fig. 3. Variantes de la chaîne opératoire de construction de valeurs caractéristiques.

ont été contestées par les représentants du monde agricole ; la définition des seuils a également été invalidée, si bien que des observations empiriques ont été mobilisées dans la réévaluation des seuils ( $c f$. Riaux, 2008). La chaîne opératoire montre que les opérations concrètes pour aller d'un objet technique à un autre ou à une décision sont plus nombreuses que celles que prévoit et organise la réglementation et qu'elles font intervenir des personnes, des interactions, des formes de savoirs et de jugements multiples. Cette forme de présentation de l'infrastructure permet ainsi de relativiser l'importance de certaines opérations, en particulier, dans ce cas, la place occupée par les activités technoscientifiques. Cette manière de restituer la chaîne d'opérations qui entre en jeu dans la prise de décision permet également une mise en visibilité des ajustements réalisés en mobilisant l'expérience directe des milieux (par l'observation); celle-ci, dans ce cas, n'apparaît plus comme une source de subjectivité, mais plutôt comme un moyen de valider ou de compléter les opérations technoscientifiques réalisées en parallèle. Ce format de description et d'analyse permet en outre d'identifier les opérations qui posent problème sur le terrain et qui se prêtent à la critique; il s'agit notamment du calcul de valeurs caractéristiques, de l'hydrométrie « instantanée » et de la définition de la valeur des seuils.

À partir de ces constats, un second niveau d'observation focalise l'attention sur les chaînes opératoires de production de données chiffrées. La figure 3, à titre d'exemple, illustre les modalités de production de valeurs caractéristiques de débit.

Comment lire ce schéma ? Soit l'objectif suivant : calculer les «valeurs caractéristiques » permettant de qualifier le régime hydrologique d'un cours d'eau, sachant que ces valeurs seront ensuite mobilisées pour déterminer les seuils, d'alerte et de crise, utiles à la décision publique. Plusieurs cas de figure peuvent se présenter, selon que l'on dispose ou non d'une chronique de données obtenue sur le site même que l'on souhaite contrôler. Si une chronique suffisante existe, on aura directement recours à un traitement statistique; sinon, la chronique pourra être complétée et/ou reconstituée, par exemple à l'aide de modèles mathématiques. Mais la valeur caractéristique pourra également être directement interpolée, en général grâce à des formules empiriques, à partir des valeurs obtenues en un autre point du cours d'eau où l'on disposait, pour des raisons souvent très contingentes, d'une chronique complète. Enfin, lorsqu'aucune donnée n'est disponible sur le cours d'eau, la grandeur caractéristique pourra être extrapolée à partir de données disponibles sur un autre cours d'eau, par exemple grâce à l'utilisation de modèles pluie-débit.

Cette forme de représentation d'une action technoscientifique présente plusieurs avantages. Cela peut tout d'abord illustrer l'étendue des possibles aux yeux mêmes des producteurs de l'infrastructure. Cela permet ensuite d'adopter un langage (relativement) simple-qui contraste avec le vocabulaire spécifique issu des mondes de la technique ou de la science - et de produire un lexique explicite pour les différentes personnes intervenant dans la construction de l'infrastructure. Il s'agit dès lors d'un outil de traduction : il permet de faire sortir des éléments spécifiquement traités par un ensemble de spécialistes des arènes scientifiques, réglementaires ou techniques ${ }^{14}$. Enfin, il donne à voir la nature des opérations et des jugements impliqués dans chacun des cheminements, ce qui peut permettre de déplacer la critique et de passer de la disqualification à la contestation. Par exemple, à propos de la construction de valeurs caractéristiques en contexte d'inexistence de données hydrologiques, mettre en question

\footnotetext{
${ }^{14}$ La notion de traduction fait référence aux approches développées en sociologie des sciences (cf. Akrich et al., 2006). De ce point de vue, l'outil proposé ici s'apparente à un objet frontière, situé à l'intersection de mondes différents et permettant de les coordonner (Star et Griesemer, 1989).
} 
les procédés d'extrapolation permet d'en expliciter le contenu. La discussion peut porter sur les conditions de pertinence et les limites de validité de cette extrapolation et non sur sa validité a priori. Ayant rendu « connaissance commune » le cheminement qui a conduit à un élément particulier de l'infrastructure technique, c'est non seulement au résultat mais à tout le processus que les acteurs pourront se référer lors de la mobilisation effective de l'infrastructure.

Enfin, un dernier intérêt de cet outil réside dans la coproduction d'une représentation de l'action technoscientifique de mesure par l'ensemble des membres du comité sécheresse. Cela fournit un moyen de construire une vision commune de la réalité ou, pour reprendre les termes de Callon et Rip (1992), une expertise partagée. Outre un déplacement de la critique, l'ouverture du processus permet d'impliquer les parties prenantes dans les choix ou dans la définition de critères pertinents pour ces choix. L'intérêt du graphisme de la chaîne opératoire est la possibilité qu'il offre d'être modifié, corrigé, enrichi. C'est ce que nous avons expérimenté en travaillant sur ces chaînes avec des hydrologues, travail qu'il faudrait approfondir et tester avec des acteurs directement en charge de la gestion des pénuries. Il pourrait s'agir a minima d'un support pour la discussion autour de l'infrastructure sociotechnique et des éléments qui la composent.

\section{Conclusion}

L'infrastructure sociotechnique de gestion des pénuries est récente, encore en cours de construction ou de stabilisation dans les départements étudiés. Bien des éléments qui la composent se prêtent à la critique des acteurs parties prenantes dans la gestion des pénuries, qu'ils soient ou non animés de mobiles stratégiques. Mais, qu'il s'agisse de contester, de contourner ou de disqualifier, la critique émise au sein des comités sécheresse porte rarement directement sur les éléments concrets qui, assemblés, font cette infrastructure. Surtout, il nous paraît important sur un plan normatif - celui d'une visée d'efficacité et de transparence de l'action publique - que l'infrastructure soit évaluée au regard de critères qui soient propres à son mode de fabrication et d'existence, celui d'une science réglementaire confrontée à la complexité du réel, dans ses dimensions à la fois biophysiques et sociopolitiques, et non au regard de critères forgés pour les situations purifiées et simplifiées de la science expérimentale de laboratoire. C'est à cette condition que l'on peut espérer parvenir à un accord sur l'état du monde, et donc engager une discussion sur les moyens de s'y adapter ou d'y remédier.

C'est ce que permet, selon nous, le concept de chaîne opératoire tel que nous nous le sommes approprié : rendre visibles l'infrastructure et les étapes de sa construction, rendre explicites les compromis et les arrangements sur lesquels elle est fondée, montrer le caractère critiquable, mais justifiable, du bricolage dont elle est nécessairement issue et ses relations aux contingences locales, sans pour autant disqualifier le résultat d'un travail collectif d'hybridation des éléments qui la composent et qui ont jalonné son adaptation aux situations qu'elle rencontre. Mobiliser la chaîne opératoire comme support de discussion en comité sécheresse pourrait alors constituer un moyen de démocratisation de l'infrastructure de l'action publique, en n'excluant pas les «profanes » de son fonctionnement, voire en les associant à son évolution, en incorporant leurs savoirs par l'identification de compétences propres à insérer dans la chaîne d'action menant à l'évaluation des situations hydrologiques. Il s'agit là d'une possibilité de dépasser la tension entre principe de justice et principe d'adaptation locale, en y impliquant l'ensemble des personnes susceptibles de mobiliser ou de réfuter cette infrastructure. Dans une perspective plus générale de démocratie technique, et à rebours des stratégies classiques de vulgarisation des sciences (Bensaude-Vincent, 2000), un tel outil de représentation pourrait rendre possible une mise en discussion des sciences respectueuse de la diversité de ses régimes épistémologiques et de ses modes concrets de fabrication.

\section{Références}

Akrich, M., Callon, M., Latour B., 2006. Sociologie de la traduction : textes fondateurs, Paris, Presses des Mines de Paris.

Balfet, H., 1991. Observer l'action technique : des chaînes opératoires, pour quoi faire?, Paris, CNRS Éditions.

Barbier, R., 2005. Le Monde du résidu. Mémoire d'habilitation à diriger des recherches, Université Paul Verlaine, Metz.

Bensaude-Vincent, B., 2000. L'Opinion publique et la science : à chacun son ignorance, Paris, Synthélabo.

Callon, M., Rip, A., 1992. Humains, non humains : morale d'une coexistence, in Theys, J., Kalaora, B., La Terre outragée : les experts sont formels, Paris, Autrement.

Creswell, R., 1996. Prométhée ou Pandore? Propos de technologie culturelle, Paris, Kimé.

Ha-Duong, M., Swart, R., Bernstein, L., Petersen, A., 2007. Uncertainty management in the IPCC: Agreeing to disagree, Global Environmental Change, 18, 1, 8-11.

Jasanoff, S., 1990. The Fifth Branch: Science Advisers as Policymakers, Cambridge (MAS), Harvard University Press.

Lascoumes, P., 1994. L'Éco-pouvoir : environnements et politiques, Paris, La Découverte.

Lascoumes, P., Le Galès, P., 2004. L'action publique saisie par ses instruments, in Lascoumes P., Le Galès, P., (Eds), Gouverner par les instruments, Paris, Presses de la Fondation nationale des sciences politiques, 11-44.

Le Bourhis, J.-P., 2004. La Publicisation des eaux : rationalité politique dans la gestion de l'eau en France (1964-2003). Thèse de doctorat en science politique, Université Paris 1 PanthéonSorbonne, Paris ; consultable en ligne à l'URL : http://tel. archives-ouvertes.fr/tel-00352536/fr/ 
Le Bourhis, J.-P., Bayet, C., 2002. Écrire le risque : étude des mécanismes d'inscription du risque inondation au niveau local. Rapport CNRS-Ministère de l'Écologie et du Développement durable (http://www.ecologie.gouv.fr/ Ecrire-le-risque-etude-des.html).

Lemonnier, P., 1980. Les Salines de l'Ouest : logique technique et logique sociale, Paris, MSH.

Leroi-Gourhan, A., 1964. Le Geste et la parole, Paris, Albin Michel.

Mermet, L., 2002. Homme ou vie sauvage? Société locale ou bureaucratie centrale? Faux dilemmes et vrais rapports de force, Annales des Mines / Responsabilité E Environnement, 28, 13-20.

Mermet, L., 2005. Prolonger l'inaction environnementale dans un monde familier : la fabrication stratégique de l'incertitude sur les ours du Béarn, Écologie E Politique, 31, 121-136.
Riaux, J., 2008. Expertise partagée et concertation imprévue pour la gestion des sécheresses, Cosmopolitiques, 17, 107-120.

Riaux, J., à paraître. Gestion de la "pénurie » d'eau en France : légitimer le bricolage par un recours à la science, in Ftaïta, T., (Ed.), Eau, société et développement durable, Paris, L'Harmattan.

Riaux, J., Barbier, R., Barreteau, O., 2009. Construire et argumenter des enjeux de vulnérabilité en comité sécheresse, in Becerra, S., Peltier, A., (Eds), Risques et environnement : recherches interdisciplinaires sur la vulnérabilité des sociétés, Paris, L'Harmattan.

Star, S.L., Griesemer, J.R., 1989. Institutional ecology, “translations" and boundary objects: Amateurs and professionals in Berkeley's Museum of Vertebrate Zoology, 1907-39, Social Studies of Science, 19, 3, 387-420. 